\title{
PARADOXICAL SPLITTING OF THE SECOND HEART SOUND
}

\author{
BY \\ IAN R. GRAY \\ From the Institute of Cardiology, London
}

Received April 20, 1955

The second heart sound is caused by closure of the aortic-and pulmonary valves at the end of ventricular systole. Two components, from the separate closure of the two valves, are usually audible because of slight asynchrony of the two ventricles, aortic closure preceding pulmonary closure in normal subjects (Leatham and Towers, 1951). Potain (1866) observed that the character of the second heart sound altered with respiration. While it might be single during the expiratory phase of continued respiration it became clearly split with inspiration. Such a change in the second sound is found in most normal subjects and is due to prolongation of right ventricular systole during inspiration. This may be explained by the increased filling of the right atrium and ventricle from the great veins which takes place with the inspiratory fall in intra-thoracic pressure. No such change can occur on the left side of the heart since intrathoracic pressure changes affect the pulmonary veins and heart chambers equally. Sometimes a clearly split second heart sound becomes single or more closely split on inspiration (Fig. 1). Such paradoxical behaviour of the second sound with respiration is due to reversal of the normal order of valve closure, pulmonary closure preceding aortic closure (Leatham, 1952). This auscultatory sign may be recognized in the neighbourhood of the pulmonary area and confirmed by phonocardiography. A study has been made of 40 cases in which paradoxical splitting of the second heart sound was recognized on clinical and phonocardiographic examination.

Method. The heart sounds were studied in simultaneous recordings taken from the pulmonary and mitral areas. The electrocardiogram and indirect carotid pulse were registered at the same time. High frequency records were used (Leatham, 1952) and measurements were made from the onset of the earliest high-frequency vibration recorded, tracings being made during held expiration. Additional phonocardiograms were made in the fourth or fifth left interspace close to the sternum in many cases to record splitting of the first heart sound, and records were taken during quiet respiration where possible. The aortic component of the second heart sound was identified in the records made at the pulmonary and mitral areas by its coincidence with the dicrotic notch of the carotid pulse (allowance being made for the delay in transmission of the arterial pulse to the carotid vessels). The pulmonary component was readily identified in records made at the pulmonary area but was absent from the mitral area records; it had no constant relation to the dicrotic notch. When splitting of the first heart sound was present the left- and right-sided components were identified where possible. These are probably caused by closure of the atrio-ventricular valves (Dock, 1933) and in normal subjects mitral closure is slightly before tricuspid. The tricuspid element is loudest at the left sternal edge in the fourth or fifth space and the mitral element at the apex. Confirmation of mitral closure was obtained from the time of onset of the carotid pulse. A measure of the duration of right and left ventricular systole could then be made from tricuspid closure to pulmonary closure and from mitral closure to aortic closure.

\section{RESULTS}

The diagnoses in the 40 cases are listed (Table I). Further sound records were studied in order to determine the incidence of paradoxical splitting of the second heart sound in these conditions. 
FIG. 1.-Paradoxical splitting of the second heart sound: effect of respiration. The sound is split by 0.04 sec. on expiration but is single on inspiration. This is due to prolongation of right ventricular systole during inspiration, the duration of left ventricular systole remaining constant throughout the respiratory cycle. (From a woman, aged 33, with a patent ductus: systemic pressure 150/62, PA pressure 115/60.)

In this and subsequent records the following abbreviations are employed. CAR =indirect carotid pulse. $\mathrm{HF}=$ high frequency. $\mathrm{LF}=$ low frequency. $\mathrm{PA}=$ pulmonary area. $\mathrm{MA}=$ mitral area. $\mathrm{LSE}=$ left sternal edge in fourth or fifth space. LD $2=$ standard lead II. 1 refers to first heart sound. 2 refers to second heart sound and letters $\mathrm{P}$ and $\mathrm{A}$ to its pulmonary and aortic components. Time markings are 0.04 and $0.2 \mathrm{sec}$.

TABLE I

Cases with Paradoxical Splitting of the Second Sound

\begin{tabular}{l|c}
\hline \multicolumn{1}{c|}{ Diagnosis } & No. \\
\hline Left bundle-branch block & 18 \\
Aortic stenosis & 10 \\
$\begin{array}{l}\text { Patent ductus arteriosus } \\
\text { Other conditions }\end{array}$ & 10 \\
& $\frac{2}{40}$ \\
\hline
\end{tabular}

Left Bundle-branch Block. The delay of aortic valve closure in left bundle-branch block was described by Wolferth and Margolies (1935) and records of the heart-sounds contributed to the correct interpretation of the electrocardiogram in conduction defects (Battro et al., 1938). Paradoxical splitting of the second heart sound was recorded in 18 out of 21 cases studied; in the other three, the heart sounds were not well enough recorded for analysis. The splitting of the second heart sound varied from 0.02 to $0.06 \mathrm{sec}$. on held expiration. The lateness of aortic valve closure was due in all cases to delay in activation of the left ventricle so that delay of mitral closure was always found where the first heart sound could be analysed (Fig. 2), and the onset of the carotid arterial pulse was correspondingly later (Fig. 3, Table II).

It is clear, therefore, that in left bundle-branch block the normal parity of the contraction times for the left and right sides is maintained. The delay of the aortic component of the second heart 


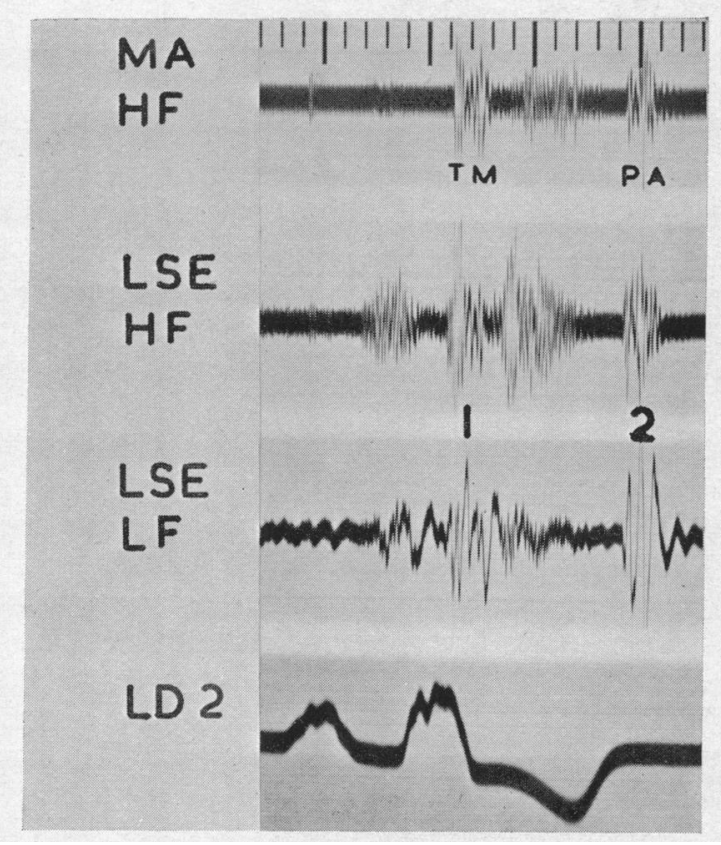

FIG. 2.-Complete left bundle-branch block. Tricuspid and mitral elements of the first heart sound are identified by the dominance of the tricuspid element in the record taken from the left sternal edge in the fourth space and of the mitral element in the trace from the apex. Mitral closure and aortic closure were further identified by their relation to the carotid pulse recorded on another tracing. There is a delay of $0.03 \mathrm{sec}$. in the onset of left ventricular contraction with a similar delay in aortic closure. (From a girl, aged 17, with mitral stenosis. QRS $0.16 \mathrm{sec}$. Record taken with held respiration.)

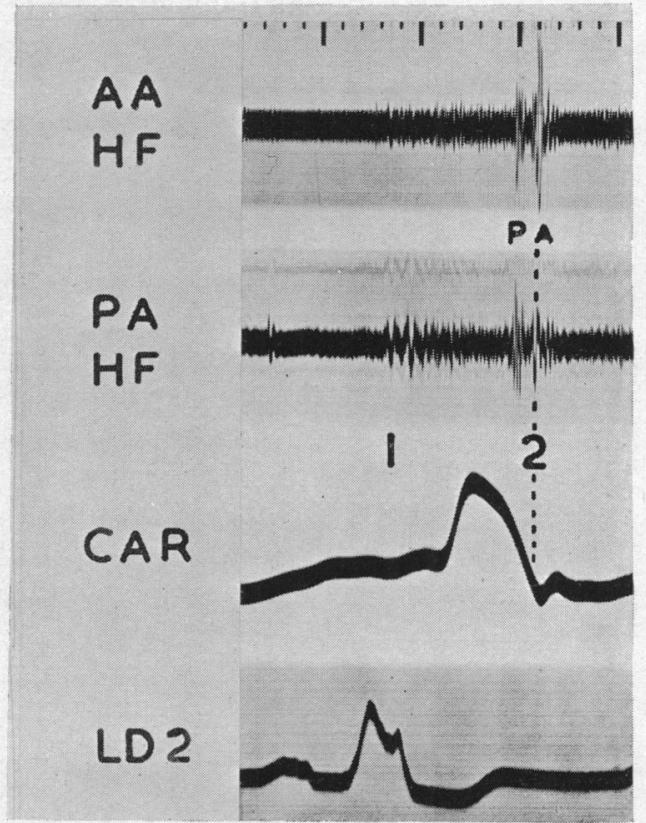

Fig. 3.-Complete left bundle-branch block, QRS 0.13 sec. Paradoxical splitting of second heart sound of 0.04 sec. A2 is recorded predominantly at the aortic area, and occurs 0.03 sec. before the dicrotic notch of the carotid trace, due to mechanical delay of the latter. (Record taken in held expiration.)

TABLE II

Timing of Cardiac Events in Left Bundle-branch Block (Mean of 18 cases)

\begin{tabular}{l|c}
\hline $\begin{array}{l}\text { Q-Mitral closure } \\
0.11 \text { sec. }( \pm 0.01) \\
\text { Normal, } 0.06 \mathrm{sec}( \pm 0.01)\end{array}$ & $\begin{array}{c}\text { R. ventricular systole (tricuspid closure-pulmonary closure) } \\
0.30 \text { sec. }( \pm 0.04)\end{array}$ \\
\hline $\begin{array}{l}\text { Q-Tricuspid closure } \\
0.07 \text { sec. }( \pm 0.02)\end{array}$ & $\begin{array}{c}\text { L. ventricular systole (mitral closure-aortic closure) } \\
0.30 \text { sec. }( \pm 0.03)\end{array}$ \\
$\begin{array}{l}\text { Normal, 0.08 sec. }( \pm 0.01) \\
\text { Q-Carotid pulse* } \\
0.16 \text { sec. }( \pm 0.02)\end{array}$ & $\begin{array}{c}\text { Pulmonary-aortic closure } \\
0.04 \text { sec. }( \pm 0.01)\end{array}$ \\
\hline
\end{tabular}

* Corrected for mechanical delay. 
sound is a delay that affects the whole of left ventricular activity. A recognizable delay of aortic closure with paradoxical splitting of the second heart sound may be regarded as an essential clinical feature of left bundle-branch block.

Aortic Stenosis. As in pulmonary stenosis where prolongation of right ventricular systole and the low pulmonary arterial pressure result in a small late pulmonary component of the second sound, so in aortic stenosis aortic closure may be delayed. In pulmonary stenosis, however, extreme stenosis with a very low pulmonary arterial pressure is compatible with life and pulmonary closure may be delayed $0.10 \mathrm{sec}$. or longer. Such severe aortic stenosis is not encountered and so, although there may be prolonged left ventricular systole with paradoxical splitting of the second heart sound, the delay in aortic closure is shorter and less constant than is the delay of pulmonary closure in pulmonary stenosis.

Paradoxical splitting of the second heart sound varying from 0.01 to $0.06 \mathrm{sec}$. was found in 10 out of a total of 23 cases of aortic stenosis studied (Fig. 4 and 5). Normal splitting was found in five and the second sound was single in eight. A single second heart sound in aortic stenosis may be due to delay in aortic closure so that it coincides with pulmonary closure, to the pulmonary component being buried in the aortic systolic murmur (Leatham, 1954), or to aortic closure being so faint as to be inaudible. Hence a single second sound in aortic stenosis is frequently associated with prolongation of left ventricular systole.

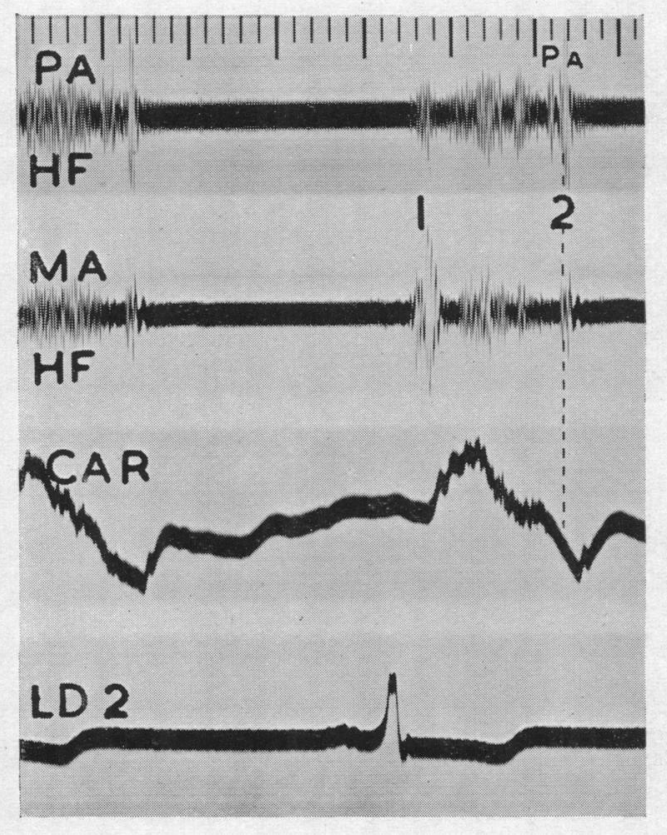

Fig. 4.-Aortic stenosis. Paradoxical splitting of second heart sounds recorded at PA. Aortic closure identified from its coincidence with dicrotic notch of carotid (mechanical delay of $0.03 \mathrm{sec}$.).

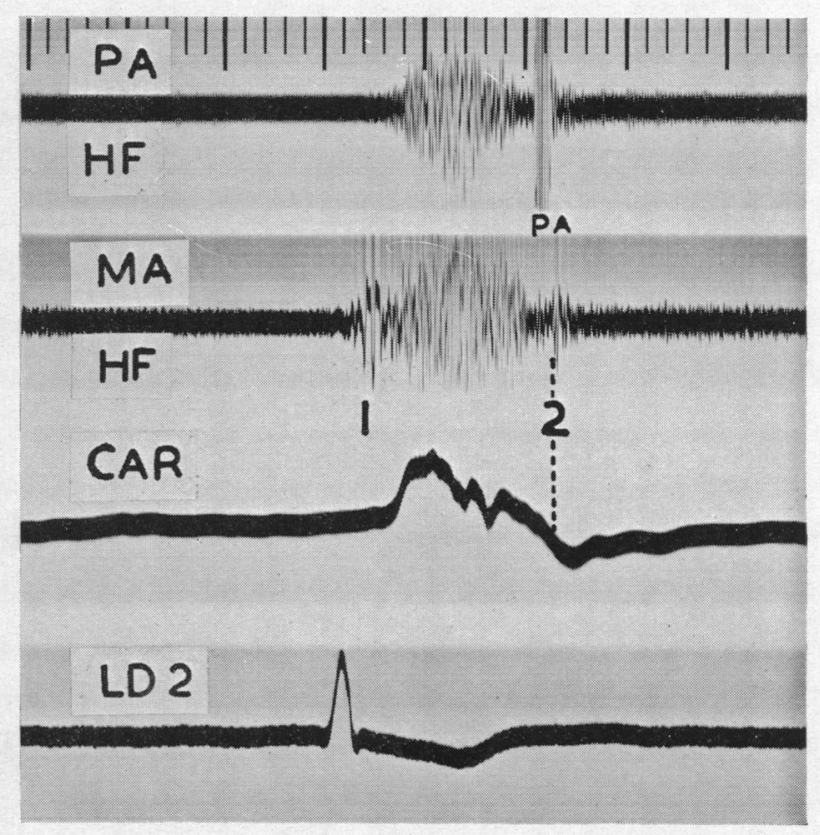

FIG. 5.-Aortic stenosis. Typical murmur is followed by paradoxical splitting of second heart sound, loud P2 in PA being followed by a small A2 (splitting is $0.04 \mathrm{sec}$.). (Record taken in held expiration.)

The timing of cardiac events in aortic stenosis with paradoxical splitting of the second sound (Table III) shows no delay in onset of left ventricular systole as judged from the times of mitral closure and upstroke of the arterial pressure pulse. Reversal of the order of aortic and pulmonary valve closure is due to left ventricular systole taking $0.04 \mathrm{sec}$. longer than right. The normal duration of ventricular systole shows considerable variation even when correction is made for the 
TABLE III

Timing of Cardiac Events in Aortic Stenosis with Paradoxical Splitting of Second Sound (Mean of 10 cases)

\begin{tabular}{l|c}
\hline $\begin{array}{c}\text { Q-Mitral closure } \\
0.07 \text { sec. }( \pm 0.03)\end{array}$ & $\begin{array}{c}\text { R. ventricular systole } \\
0.29 \text { sec }( \pm 0.04): 0.32 \text { sec.* }\end{array}$ \\
\hline $\begin{array}{c}\text { Q-Tricuspid closure } \\
0.08 \text { sec. }( \pm 0.03)\end{array}$ & $\begin{array}{c}\text { L. ventricular systole } \\
0.33 \text { sec. }( \pm 0.03): 0.36 \text { sec.* }\end{array}$ \\
\hline $\begin{array}{c}\text { Q-Carotid pulse } \\
0.10 \text { sec. }( \pm 0.03)\end{array}$ & $\begin{array}{c}\text { Pulmonary-aortic closure } \\
0.04 \text { sec. }( \pm 0.02)\end{array}$ \\
\hline
\end{tabular}

* Corrected to cycle length $1 \cdot 0 \mathrm{sec}$. using formula: Corrected time $=$ Measured time $\div \sqrt{ }$ Cycle length (sec.) (Bazett, 1920).

heart rate. Mean left ventricular systole measured in 20 healthy subjects was $0.34 \mathrm{sec} .( \pm 0.02)$ when corrected for cycle length of $1.0 \mathrm{sec}$. Measurement of right ventricular systole during held expiration showed, however, that the durations of systole of the right and left sides in normal subjects were nearly always identical, mean right ventricular systole in this group also being 0.34 sec. $( \pm 0.02)$. Comparison of the duration of systole in aortic stenosis with paradoxical splitting of the second sound with the normal shows prolongation of left ventricular systole $(0.36 \mathrm{sec}$.) and shortening of right ventricular systole $(0.32 \mathrm{sec}$.). The former has been shown to be a feature of severe aortic stenosis (Katz and Feil, 1925) and is a result of obstruction to left ventricular outflow: the latter is difficult to explain but may be partly due to reduced systemic venous return.

The aortic stenosis was regarded as severe on other clinical grounds in eight of the ten cases in which paradoxical splitting was found. Normal splitting of the second sound was never found when the aortic stenosis was severe.

Patent Ductus Arteriosus. Paradoxical splitting of the second heart sound varying from $0.01 \mathrm{sec}$. to $0.07 \mathrm{sec}$. was found in 10 out of 29 cases of patent ductus (Fig. 1 and 6). The pulmonary component of the second sound was obscured by the murmur in three cases which had to be rejected, and of the remaining 16, splitting was normal in nine and the second sound was single in seven.

The paradoxical splitting is due, as in aortic stenosis, to a disparity between the duration of right and left ventricular systole. When these are corrected for heart rate in cases of patent ductus with paradoxical splitting there is found to be prolongation of left ventricular and shortening of right ventricular systole. The former change suggests increase in the diastolic size of the left ventricle (Wiggers, 1949), associated in patent ductus with overfilling from the excessive pulmonary flow. The shortened right ventricular systole may be due in part to reduced systemic venous return and possibly also to increased pulmonary diastolic pressure.

TABLE IV

Timing of Cardiac Events in Patent Ductus with Paradoxical Splitting of Second Sound (Mean of 10 cases)

\begin{tabular}{l|c}
\hline $\begin{array}{c}\text { Q-Mitral closure } \\
0.06 \text { sec. }( \pm 0.01)\end{array}$ & $\begin{array}{c}\text { R. ventricular systole } \\
0.27 \text { sec. }( \pm 0.04): 0.31 \text { sec.* }\end{array}$ \\
\hline $\begin{array}{c}\text { Q-Tricuspid closure } \\
0.07 \text { sec. }( \pm 0.02)\end{array}$ & $\begin{array}{c}\text { L. ventricular systole } \\
0.31 \text { sec. }( \pm 0.04): 0.35 \text { sec.* }\end{array}$ \\
\hline $\begin{array}{c}\text { Q-Carotid pulse } \\
0.10 \text { sec. }( \pm 0.01)\end{array}$ & $\begin{array}{c}\text { Pulmonary-aortic closure } \\
0.03 \text { sec. }( \pm 0.02)\end{array}$ \\
\hline
\end{tabular}

* Corrected for cycle length $1 \cdot 0 \mathrm{sec}$. 
Great variation is now recognized in the clinical and hæmodynamic findings in patent ductus according to the size of the communication and the pulmonary vascular resistance. The volume of the shunt and its direction might be expected to influence the second heart sound. Paradoxical splitting of the second sound was found in more than half the cases in which the shunt was from left to right ( 8 out of 15). It was found in only 2 out of 11 cases with severe pulmonary hypertension and balanced or reversed shunt. Measurements of pulmonary arterial pressure and pulmonary and systemic flow (Table V) suggested that when the pulmonary vascular resistance was low and the shunt small so that the pulmonary arterial pressure remained normal, the normal order of valve closure obtained. When the shunt was of greater magnitude and the pulmonary arterial pressure

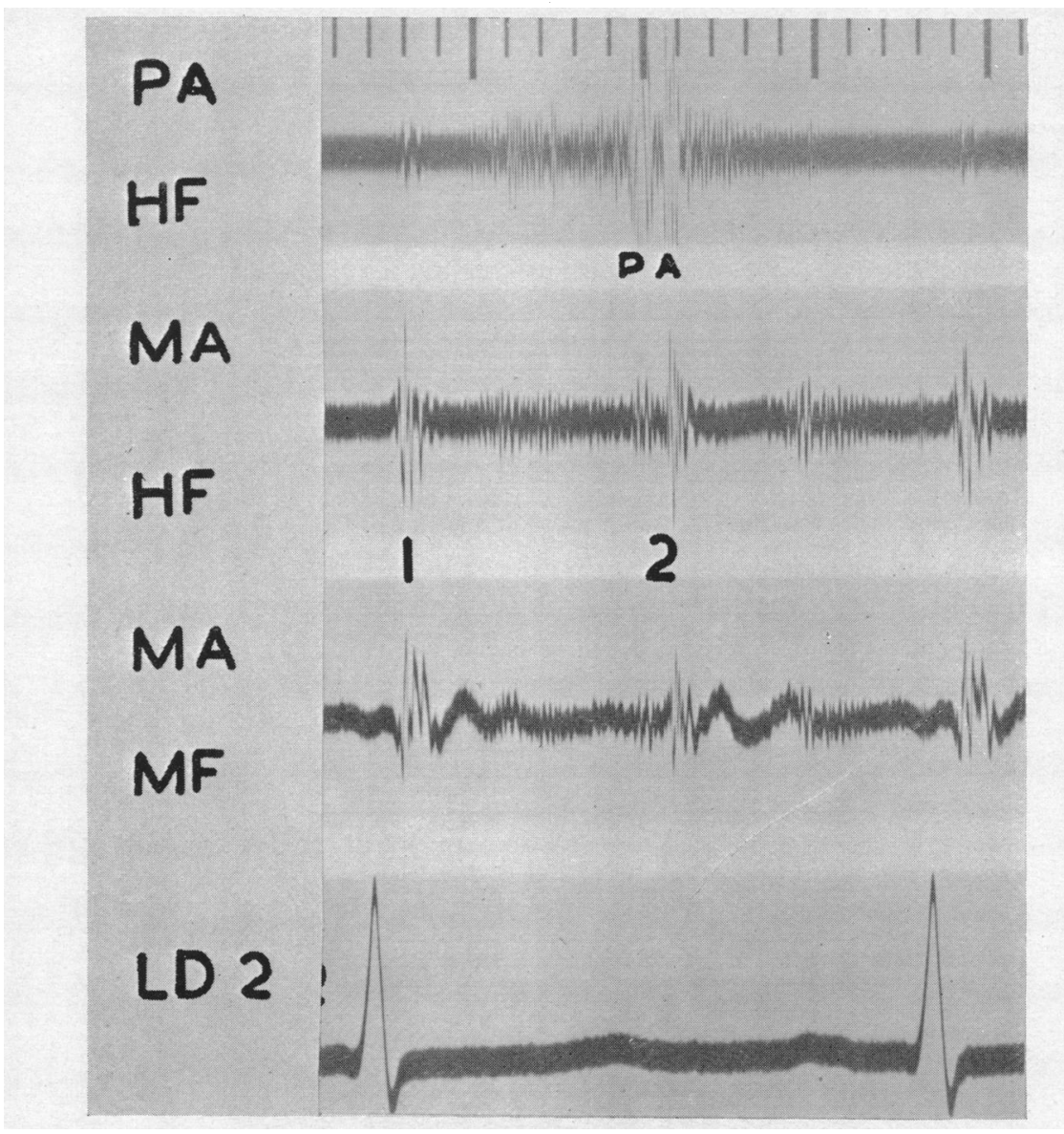

FIG. 6.-Patent ductus arteriosus. Continuous murmur recorded at PA. First heart sound is single. Paradoxical splitting of second heart sound recorded, A2 being identified by its presence at the MA and its relation to the dicrotic notch recorded on another tracing.

moderately increased paradoxical splitting commonly occurred. When the resistance was greatly increased so that there was no longer a left-to-right shunt the second sound was frequently single.

Other Cases. Paradoxical splitting of the second heart sound was heard and recorded in two further cases, one of hypertensive heart disease and one of mitral stenosis. Phonocardiograms of 10 other examples of each of these conditions were studied, in all of which the normal order of aortic and pulmonary valve closure was found. Paradoxical splitting of the second sound may therefore be regarded as exceptional in these conditions. 


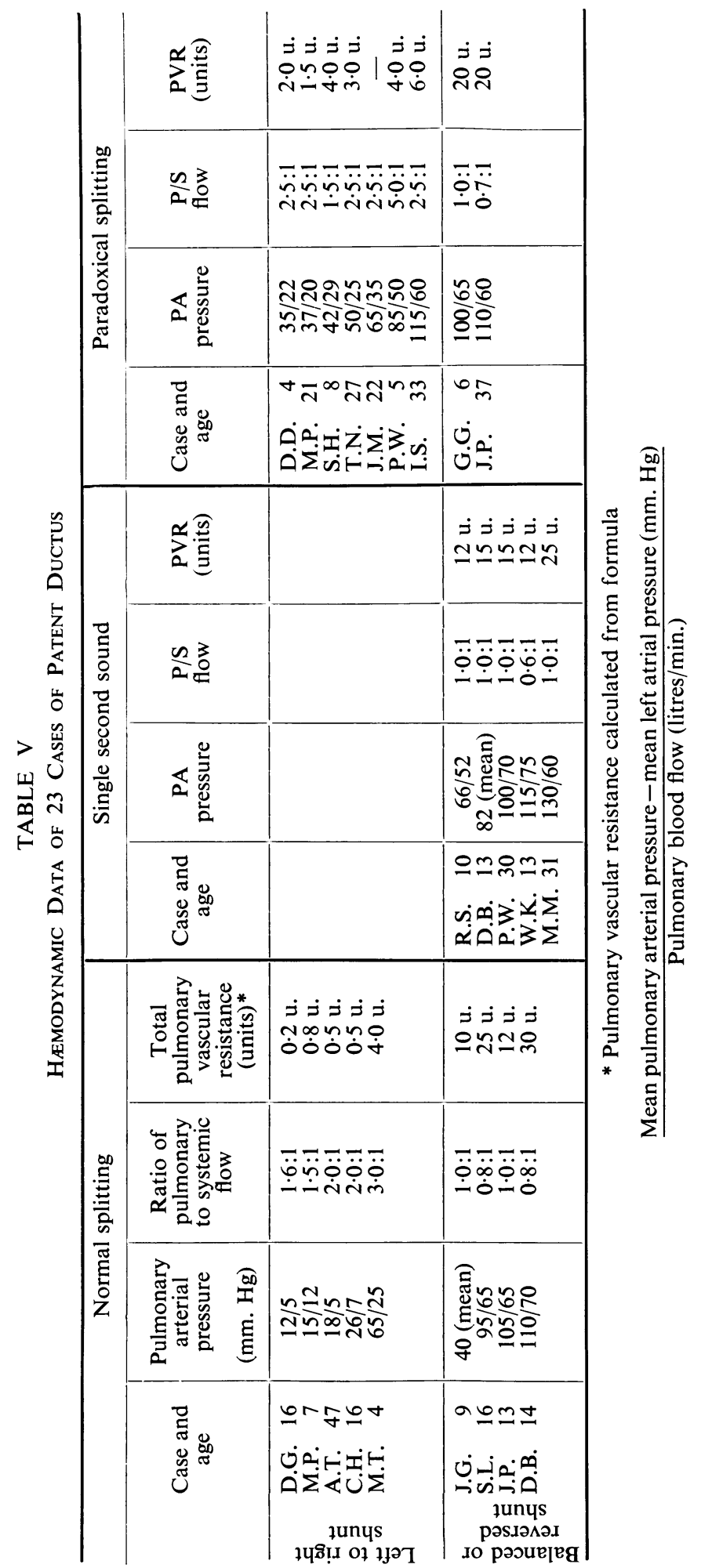




\section{Discussion}

Careful auscultation over the base of the heart is necessary if paradoxical splitting of the second sound is to be recognized. Both components of the sound are generally best heard in the second left intercostal space but it is often easier to listen to them throughout the respiratory cycle in the third or even fourth space. The sign may escape recognition if respiration is noisy or if one of the components of the second sound is faint. Phonocardiography is of considerable value in these doubtful cases.

The sign was easily recognized in left bundle-branch block and it is possible to distinguish this from right branch block and from other causes of a widely split second sound with reasonable accuracy at the bedside. In aortic stenosis, the assessment of which may be difficult when mitral valve disease co-exists, the presence of a normally split second sound may be regarded as strong evidence against severe aortic stenosis, which should always be accompanied by a single or a paradoxically split second sound.

The presence of paradoxical splitting of the second sound in many cases of patent ductus is particularly interesting. This sign has not been found in any other form of left-to-right shunt. In doubtful cases it has proved a useful sign in establishing a clinical diagnosis. Its presence suggests a substantial left-to-right shunt with some elevation of pulmonary arterial pressure.

\section{SUMMARY}

Reversal of the normal order of aortic and pulmonary valve closure may be recognized clinically by the closing up of a split second heart sound on inspiration and has been called paradoxical splitting of the second sound. This has been studied by phonocardiograms of 40 cases.

It was invariably found with left bundle-branch block and was due to delay in the onset of left ventricular systole.

It was found in 10 out of 23 cases of aortic stenosis. It was due to prolongation of left ventricular systole in the presence of a shortened right ventricular systole and was generally a sign of severe stenosis.

It was found in 10 out of 29 cases of patent ductus. This also was due to prolongation of left ventricular and shortening of right ventricular systole. It was usually found in cases with a substantial left-to-right shunt and a moderate increase of the pulmonary arterial pressure.

The mechanism of paradoxical splitting of the second heart sound is discussed and its value in bedside diagnosis assessed.

I wish to thank Dr. Aubrey Leatham for much help and encouragement in the preparation of this paper and the Physicians of the National Heart Hospital for permission to study patients under their care.

\section{REFERENCES}

Battro, A., Braun Menendez, E., and Orias, O. (1938). Arch. Mal. Coeur, 31, 250.

Bazett, H. C. (1920). Heart, 7, 393.

Dock, W. (1933). Arch. intern. Med., 51, 737.

Katz, L. N., and Feil, H. S. (1925). Heart, 12, 171.

Leatham, A. (1952). Brit. med. Bull., 8, 333.

- and Towers, M. (1951). Brit. Heart J., 13, 575.

Potain, C. (1866). Bull. Soc. Méd. Hôp. Paris, 3, 138.

Wiggers, C. J. (1949). Physiology in Health and Disease, p. 679. London.

Wolferth, C. C., and Margolies, A. (1935). Amer. Heart J., 10, 425. 